\title{
HEPATITE AGUDA FULMINANTE E TIREOTOXICOSE: RELATO DE CASO
}

\author{
Hepatitis acute fulminant and Thyrotosicosis: Case Report
}

\author{
Carla Paixão Miranda
}

\section{RESUMO}

Paciente feminino, 44 anos transferida para o centro de terapia intensiva (CTI) do Hospital das Clínicas de Belo Horizonte/MG com quadro de dor abdominal, náuseas, vômitos, icterícia e febre, anorexia, inapetência iniciado há 30 dias. História de hipotireoidismo e tratamento irregular com T4 há cinco anos; interrompeu a medicação quando iniciou o quadro atual. Apresentava T3 $(7,26 \mathrm{pg} / \mathrm{ml})$ VN.: 2,77 a 5,07; TSH $(0,214 \mathrm{mU} / \mathrm{L}) \mathrm{VN}: 0,465$. Transaminases AST (977 U/L) VN.:15 a 46; ALT (795 U/L) VN.:13 a 69; bilirrubina total (4.12 mg/dL) VN.: 0.2 a 1.3; bilirrubina direta $(30 \mathrm{mg} / \mathrm{dL}) \mathrm{VN}$.: até 0.25 . Foi mantida sem anti-tireoidiano e iniciado propranolol até dose de $480 \mathrm{mg} / \mathrm{dia}$. Exame de ressonância magnética abdominal e colangio com líquido livre abdominal, sugestivo de estar relacionado ao processo inflamatório. Espessamento inespecífico da vesícula biliar. Derrame pleural bilateral. Sorologia para vírus da hepatite A, B e C negativo. Ocorreu piora da função hepática e manutenção dos níveis elevados de hormônios tireoidianos. A paciente evoluiu para um quadro de hepatite aguda fulminante, imediatamente submetida ao transplante hepático. Após procedimento, o fígado (explante) foi enviado para estudo anatomopatológico; os achados histopatológicos incluíram presença de extensa área de necrose do parênquima hepático. Nos septos remanescentes, mostravam-se infiltrados inflamatórios mononucleares (IIMM). Conclui-se, portanto, que o hipertireoidismo crônico pode exacerbar e perpetuar a disfunção hepática aguda fulminante e que o tratamento medicamentoso regular aliado aos exames laboratoriais de rotina clínica poderão prevenir complicações graves, permitindo uma terapêutica bem sucedida em pacientes com hipertireoidismo.

Descritores: Tireoide; Hepatite; Tireotoxicose; Fígado; Inflamação.

\section{Institution}

Programa de Pós-Graduação Ciências da Saúde: Infectologia e Medicina Tropical, Faculdade de Medicina da UFMG; Ambulatório de Referência em Doenças Infecto-Parasitarias CTR-DIP - Hospital das Clínicas da Universidade Federal de Minas Gerais - Belo Horizonte/MG

\section{Correspondência:}

Carla Paixão Miranda

Rua. Campos Elíseos, 196 - 2 andar - CEP 30431-068 -

Belo Horizonte/MG

Telefone: (38) 99157-7523

E-mail: carlamedicina@ufmg.br

Recebido em: 19/08/2016

Aceito em: 20/09/2016

\section{APRESENTAÇÃO DO CASO}

Paciente 44 anos, feminino, desenvolveu tireotoxicose com acometimento hepático, com evolução para quadro de hepatite aguda fulminante, após cinco anos de terapia com uso de levotiroxina (T4L) $75 \mathrm{mcg} /$ dia, prescrito para o hipotireoidismo. Paciente havia interrompido medicação antitireoidiana (levotiroxina $75 \mathrm{mcg} / \mathrm{dia}$ ) há, aproximadamente, duas semanas antes da instalação do quadro. Iniciou com mal estar, náuseas, anorexia, inapetência e icterícia, 30 dias antes da instalação do quadro de hepatite fulminante. Iniciou acompanhamento na cidade de Paracatu. Quatro dias após a internação, ao realizar US ABD superior: fígado pouco aumentado; contornos lisos e regulares. Aumento difuso da ecogenicidade vias biliares intra-hepáticas de paredes espessadas, sem dilatação. Vias biliares extra-hepáticas sem dilatação. 
Colédoco = $3 \mathrm{MM}$. Ao exame de ressonância magnética abdominal e colangiorresonância. Sete dias após a internação, foi transferida para o CTI de Patos de Minas devido à piora neurológica (RNM ECG 6). Exames evidenciaram insuficiência hepática aguda: RNI incoagulável; BT > 20. Apresentou TGO/TGP > 1000 em uma amostra; mantendo, posteriormente, TGO/TGP, aproximadamente 300/400. Nove dias após internação, foi realizada ressonância magnética abdominal e colangioressonância, evidenciando presença de líquido livre abdominal, sugestivo de estar relacionado ao processo inflamatório. Espessamento inespecífico da vesícula biliar. Vias biliares e duto pancreático de calibre e trajeto normais. Parênquima hepático heterogêneo. Derrame pleural e bilateral. Exame de tomografia computadorizada sem evidências de alterações. Os exames laboratoriais: anti HCV negativo; anti HAV IgM negativo; HBsAg negativo; HIV negativo; VDRL negativo. Aos exames laboratoriais: Plaquetas $=119.000 / \mathrm{mm} 3$ (faixa de referência - VR.: 150-450 x 103); Hemoglobina $=9,4 \mathrm{~g} / \mathrm{dL}$ (faixa referência - VD.: 12,0 a $16,0 \mathrm{~g} / \mathrm{dL}$ ); Hematócrito $=28,7 \%$ (faixa de referência - VR.: 36 a $46 \%$ ); Globulinas $=5,780 \mathrm{~g} / \mathrm{dL}$ (faixa de referência VD.: $2,0-3,9$ ) RNI $=5,59$ (faixa de referência - VD.:2,03,0 ); Ureia $=15 \mathrm{mg} / \mathrm{dL}$ (faixa de referência - VD.: 16-40 $\mathrm{mg} / \mathrm{dL}$ ); Creatinina $=0,9 \mathrm{mg} / \mathrm{dL}$ (faixa de referência VD.: 0,6-1,2 mg/dL); TGO= $271 \mathrm{u} / \mathrm{l}$ (faixa de referência - VD.: 5,0-40), TGP = $189 \mathrm{U} / \mathrm{L}$ (faixa de referência VD.:7,0-56); gama GT $=41 \mathrm{U} / \mathrm{L}$ (faixa de referência VD.:0,5-36); $B T=22,7$ (faixa de referência - VD.: 0,3$1,3 \mathrm{mg} / \mathrm{dL}$ (5-17 mmol/L SI); $\mathrm{Na}+; 145 \mathrm{mmol} / \mathrm{L}$ (faixa de referência - VD.: 138-142); $\mathrm{K}+3,2 \mathrm{mmol} / \mathrm{L}$ (faixa de referência - VD.: 3.8 a 5.5); $\mathrm{TSH}=0,214 \mu \mathrm{UI} / \mathrm{mL}$ (faixa de referência - VD.: 0,465 a 4,680); T3 $=7,26 \mathrm{pg} / \mathrm{ml}$ (faixa de referência - VD.: 2,77 a 5,27); T4 $=0,12 \mathrm{ng} / \mathrm{dL}$ (faixa de referência - VD.: 0,78 a 2,19). Onze dias após a internação, paciente foi transferida para o Hospital das Clínicas de Belo Horizonte, chegando entubada. Icterícia $(+++/ 4+)$. Abertura ocular, sem contato. Estabilidade hemodinâmica. Ao passar pela avaliação da neurologia: paciente apresentava quadro sugestivo de encefalopatia metabólica, sem sinais clínicos ou radiológicos de lesão do SNC. Foi mantida sem o antitireoidiano, devido ao risco de piora da lesão hepática, aumentado propranolol até a dose de $480 \mathrm{mg} / \mathrm{dia}$. Tendo em vista a piora do quadro, agravamento da diarreia, emagrecimento de oito $\mathrm{kg}$ em 15 dias, necessitando inclusive de nutrição enteral. Exames laboratoriais pré-transplante (Tabela 1). Em razão da anemia, foi submetida à hemotransfusão. Ocorreu piora da função hepática $(\mathrm{TAP}=67 \%$; AST $=$

Tabela 1: Sumário de exames laboratoriais

\begin{tabular}{|c|c|c|c|}
\hline Exames & Internação & Evolução & VN \\
\hline Bilirrubina total (mg/dL) & 4,12 & 3,9 & 0.2 a 1.3 \\
\hline Bilirrubina direta (mg/dL) & 5,6 & 2,4 & até 0.25 \\
\hline Fosfatase Alc (U/dL) & 220 & 84 & $38-126$ \\
\hline Gama GT (U/L) & 192 & 56 & $12-43$ \\
\hline Albumina $(\mathrm{g} / \mathrm{dL})$ & 1,8 & 2,5 & $3,4-5,0$ \\
\hline Glicose (mg/dL) & 174 & 126 & $55-99$ \\
\hline Ureia (mg/dL) & 15 & 11 & $15-45$ \\
\hline Creatinina (mg/dL) & 0,9 & 1,21 & 0,6 a 1,3 \\
\hline Sódio (mmol/L) & 145 & 149 & $135-145$ \\
\hline Potássio (mmol/L) & 3,5 & 3,2 & $3,5-5,5$ \\
\hline Hemácias milhões/ $\mu \mathrm{L}$ & 2,45 & 4,1 & $4,5-5,5$ \\
\hline Hemoglobina g/dL & 7,2 & 9,6 & $13,0-17,5$ \\
\hline Hematócrito (\%) & $20,7 \%$ & 28,7 & $40-50 \%$ \\
\hline 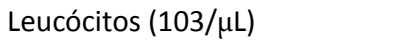 & 5,2 & 8,9 & $4,0-11,0$ \\
\hline Basófilos & 0 & 0 & $0-1$ \\
\hline Eosinófilos (\%) & 1,2 & 0,7 & $1-5$ \\
\hline Segmentados (\%) & 36 & 61 & $40-55$ \\
\hline Linfócitos (\%) & 44 & 62 & $40-55$ \\
\hline Monócitos (\%) & 21 & 12 & $2-10$ \\
\hline Plaquetas $103 / \mu \mathrm{L}$ & 119 & 10800 & $150-450$ \\
\hline TAP (\%) & 98 & 67 & $10,7-14,3$ \\
\hline
\end{tabular}

$V N=$ Valores normais; $\quad T A P=$ Tempo de atividade de protrombina 
990U/L e $A L T=1.007 \mathrm{U} / \mathrm{L})$, mantendo níveis elevados de hormônios tireoidianos ( $\mathrm{T} 4=28,7 \mathrm{ng} / \mathrm{mL}$ e T3 $=20$ $\mathrm{pg} / \mathrm{mL}$ (faixa de referência - VD.: 2.7 a 5.27) (Figura 1). Paciente foi submetida ao transplante hepático com urgência. Apresentou PA elevada, tendo recebido manitol e furosemida no início do procedimento.

As dosagens de T4L, T3L das transaminases nos períodos da internação, cirurgia e pós-operatório podem ser vistas nas figuras 1, 2 e 3 .

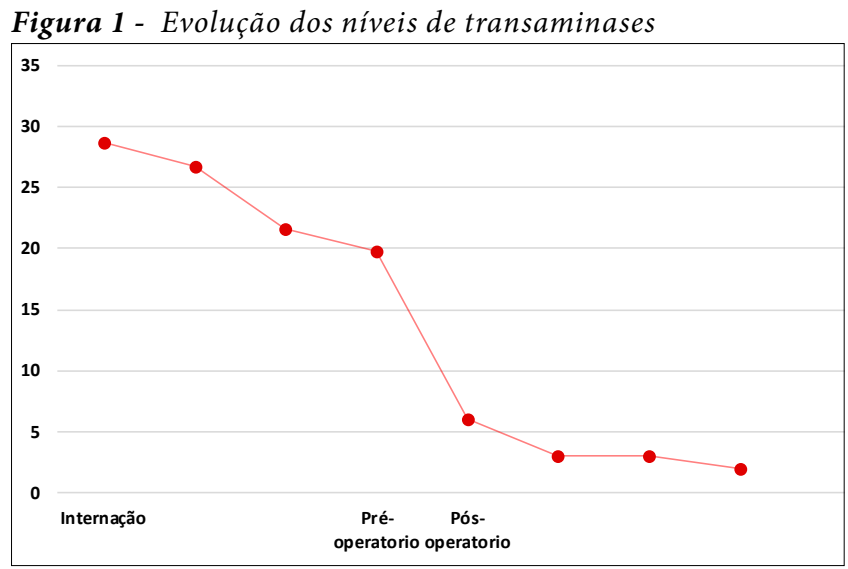

$T 4 L=$ tiroxina livre, valores normais: 0,78 a 2,19 $\mathrm{ng} / \mathrm{dL}$

Figura 2 - Evolução dos níveis de T3L

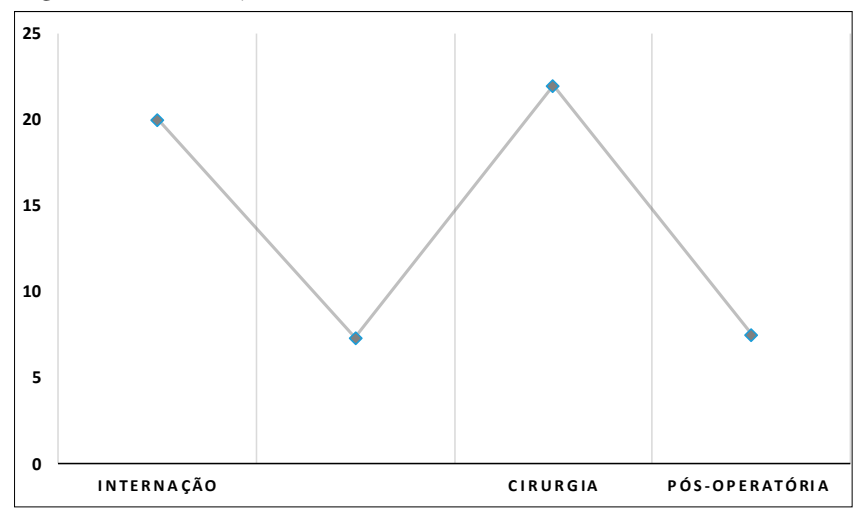

$T 3 L=$ Triodotironina livre, valores normais: 2,77 a 5,27 pg $/ \mathrm{mL}$

Figura 3 - Evolução dos níveis de transaminases

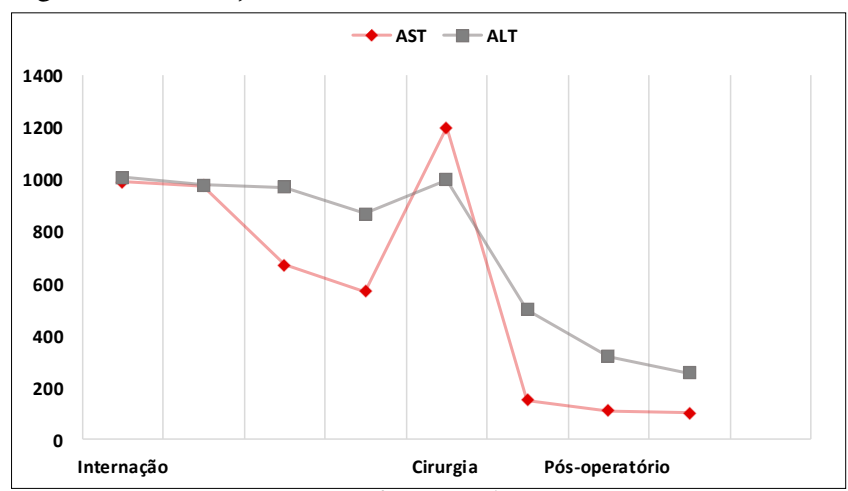

AST = aspartato aminotransferase, valores normais: 10 a $34 \mathrm{U} / \mathrm{L}$; $A L T=$ alanina aminotransferase, valores normais: 9 a $45 \mathrm{U} / L$.

\section{ESTUDO HISTOPATOLÓGICO DO FIGADO}

Os achados que caracterizam a hepatite aguda fulminante por tireotoxicose medicamentosa nos cortes histológicos do fígado incluem arquitetura lobular subvertida, pela presença de extensas áreas de necrose do parênquima hepático, ora por confluências de necrose focais formando porres que unem estruturas vasculares, portais e centrolobulares entre si, em permeio à área de hemorragia. Nos septos remanescentes, mostramse infiltrados inflamatórios mononucleares (IIMM) que ultrapassam a placa limitante de hepatócitos. No parênquima, observam-se depósitos dos pigmentos de hemossiderina e degeneração hidrópica dos hepatócitos. Conclui-se, portanto, que o diagnóstico é de hepatite aguda fulminante medicamentosa com necrose maciça, segundo relação entre os dados clínicos e o exame histopatológico.

\section{DISCUSSÃO}

Relatamos o caso de uma paciente com disfunção tireoidiana com evolução para hepatite aguda fulminante associada. A paciente foi submetida com urgência ao transplante hepático.

O hormônio tireoidiano (HT) apresenta influência na função hepática. Hepatócitos são células altamente responsivas ao HT que apresentam a maioria dos seus receptores para TSH localizados no meio intranuclear. ${ }^{1}$ $\mathrm{O}$ HT modula a atividade de várias organelas dentro dos hepatócitos, evidenciando-se sua ação nas mitocôndrias, consumo de oxigênio e fosforilação oxidativa elevadas na patogênese da hiper-estimulação tireoidiana. ${ }^{2,3}$ Para a maioria dos pacientes com hiperestimulação da glândula tireoide, a ocorrência de disfunção hepática acontece em virtude dos efeitos metabólicos provenientes do excesso de HT, em concomitância à hipóxia tecidual resultante do aumento no consumo de oxigênio esplânico com consequente, elevação da captação de oxigênio hepático. ${ }^{4}$

A lesão hepática associada ao hipertireoidismo pode variar desde leves alterações das enzimas hepáticas até grave alterações histopatológicas pouco especificas com necrose hepatocelular. ${ }^{5}$ Estudos realizados por Sola J et al., (1991) corroboram com nossos achados, ao encontrar correlação positiva entre as concentrações bioquímicas dos hormônios tireoidianos em relação às alterações histopatológicas. ${ }^{5}$ Quanto aos sinais clínicos, o mesmo estudo relatou hepatomegalia a uma frequência de até $33 \%$ e de icterícia entre 5,3 a $50 \%$., As alterações bioquímicas para os testes da função hepática em pacientes com tireotoxicose medicamentosa 
é bastante variável, em média, com prevalência de cerca de $75 \%$, podendo variar entre $15 \%$ e $90 \% .^{8,9} \mathrm{Na}$ análise laboratorial, destaca-se elevação mais frequente na gama GT e fosfatase alcalina. ${ }^{10,11,12}$ Huang $M-J$, et al., (1994) ao avaliarem 95 pacientes com hipertireoidismo, sem alterações cardiovasculares e com testes negativos para hepatites virais, observaram maior frequência de elevação nas concentrações séricas de AST e ALT, $28,4 \%$ e $38,3 \%$, respectivamente. Fong et al., (1992) demostraram elevação nos níveis séricos de ALT em $67 \%$ dos casos de hipertireoidismo analisados 10 . Como demonstrado em vários estudos, as enzimas hepáticas tendem a se normalizar na maioria dos pacientes, com restauração do estado eutireoidiano, sugerindo que a elevação dos níveis de AST e ALT ocorre por hiperestimulação da glândula tireoide..$^{11,12}$

O uso irregular de terapias antitireoidianas é tida como causa para $\mathrm{o}$ acometimento hepático em pacientes com hipertireoidismo.111 Elevações transitórias e assintomáticas nas concentrações de AST e gama GT foram descritas por Huang et al.; $33 \%$ dos pacientes em uso de PTU (propiltiouracil) desenvolveram lesões hepáticas leves, não necessitando de suspensão da terapia..$^{13}$ A incidência de pacientes que apresentam necrose hepática submaciça atribuída a tireotoxicose é de cerca de $20 \% .^{13}$ No entanto, a maioria dos pacientes recupera com a retirada da PTU. Ao se avaliar o banco de dados de transplante hepático na população americana, entre os anos de 1990 e 2002, foi mostrado que, excluindo-se pacientes em uso de acetoaminofem, o PTU era a segunda droga relacionada às lesões hepáticas com urgência para transplante, representando $9,5 \%$ dos casos. ${ }^{14,15}$ Diversos estudos sugerem que o mecanismo de ação pelo qual a propiltiouracil (PTU) causa lesão aos hepatócitos são toxicidade direta ou reação idiossincrática..$^{14,15}$

A tabela 2 apresenta alguns estudos de maior relevância sobre a utilização de transplante hepático por toxicidade medicamentosa.

Tabela 2: Estudos de maior relevância sobre transplante hepático por hepatotoxicidade medicamentosa.

\section{Estudo e ano}

\begin{abstract}
Liver Transplantation for Acute Liver From Drug Induced Liver Injury in The United States.
\end{abstract}

Russo M.W et al., (2004).
Utilizou-se uma base de dados de transplantes hepáticos de uma população dos EUA para, identificar a taxa de transplante de fígado devido a hepatotoxicidade medicamentosa.

Notificaram-se 6 casos de insuficiência hepática fulminante após complicação grave de tempestade tireoidiana os pacientes foram submetidos a tireodoctomia seguido de transplante hepático.

Rangnerkar A.S et al., (2013).

Quatro fármacos foram implicados em $42 \%$ dos transplante de fígado entre eles estão: isoniazida, Propiltiouracilo, fenitoína e valproato. O percentual de sobrevivência após um ano do enxerto para toda a coorte foi de 77 e $71 \%$.

Os pacientes passaram por tireodoctomia seguido de transplante hepático. Os níveis séricos dos hormônios estimulantes da tireoide triiodotironina, tiroxina e transaminases normalizaram. 10 após o transplante hepático e a tireodoctomia os pacientes estavam aptos à alta hospitalar.

\author{
Notificaram-se 6 casos de insuficiência \\ hepática fulminante após complicação grave \\ de tempestade tireoidiana os pacientes foram \\ submetidos a tireodoctomia seguido de \\ transplante hepático. \\ Thyroid storm complicated by fulminant \\ hepatic failure: case report and \\ literature review. \\ Hambleton Cet al., (2013).
}

Os pacientes passaram por tireodoctomia seguido de transplante hepático. Os níveis séricos dos hormônios estimulantes da tireoide triiodotironina, tiroxina e transaminases normalizaram. 10 após o transplante hepático e a tireodoctomia os pacientes estavam aptos à alta hospitalar.
No presente relato de caso, acredita-se que o hipertireoidismo crônico não compensado esteve associado ao uso irregular da medicação antitireoidiana, com base nas avaliações laboratoriais, clínicas e anatopatológicas, sugerindo estresse metabólico e toxicidade do fígado. Essa associação predispôs ao curso da hepatite aguda fulminante por toxicidade medicamentosa. ${ }^{14,15}$

As terapias para doença de Graves visam a inibição da síntese dos hormônio tireoidianos, destruição ou remoção do tecido da tireoide, radioiodo ou cirurgia. A embolização arterial de tecido tireoidiano mostrou-se eficaz, mas sua aplicação é pouco difundida, havendo poucos estudos exploratórios. Ainda não se sabe a melhor terapia para a doença de Graves, tendo em vista que a maioria dos estudos são inconclusivos e paradoxais..$^{16,17}$

As drogas antitireoidianas ainda são a primeira escolha para o tratamento de pacientes com hipertireoidismo moderado, bócio pequeno, oftalmopatia ativa. 
Pacientes com contraindicação aos antitireoidianos ou que apresentem menores chances de remissão (bócio volumoso, hipertireoidismo grave com níveis muito elevados de T3 e T4 e relação T3/ T4 > 20), têm sido indicados em sua maioria ao tratamento com radioiodo como primeira opção.18 $\mathrm{O}$ cenário atual tem estabelecido que intervenções cirúrgicas são bastante limitadas principalmente em crianças e gestantes com efeitos colaterais, não aderência ou não resposta da tireotoxicose às drogas antitireoidianas, bócio volumoso com suspeita de doença maligna associada. Outra vantagem da cirurgia é o restabelecimento imediato do eutireoidismo. ${ }^{18}$

Com base nas premissas que norteiam o caso descrito, o controle da tireotoxicose não foi possível, devido à rápida proporção e gravidade com que a doença evoluiu. A suspensão da medicação poderia ter levado ao controle da tireotoxicose com rápida normalização das enzimas hepáticas e da função hepática. Esse controle é mais rapidamente obtido com tireoidectomia. Outro tratamento bastante citado para pacientes com tireotoxicose é a plasmaférese utilizada há vários anos, tendo sido empregada tanto em crise tireotóxica quanto em agrunulocitose, vasculite, hepatoxicidade e outros efeitos adversos induzidos por PTU ou metimazol.
Após transplante hepático, não tivemos mais acesso ao caso, portanto, não sabemos sobre o controle endócrinometabólico e ajustes terapêuticos. É importante ressaltar que a equipe médica assistente deve estar ciente que a paciente poderá necessitar de rigoroso controle de medicamentos, tendo em vista que ela pode ter elevada concentração de tiroxina na circulação.

Pacientes que evoluíram com quadro de insuficiência hepática aguda por intoxicação medicamentosa apresentam altas taxas de morbidade e mortalidade, podendo apresentar complicações no enxerto como disfunções, rejeição, trombose, sangramento ou fuga biliar. $^{19,20}$

\section{CONCLUSÃO}

Conclui-se, portanto, que pacientes com hipertireoidismo não compensado em uso irregular da medicação antitireoidiana podem vir a desenvolver disfunção hepática exacerbadas, por efeito tóxico desta classe de medicamento. Recomenda-se a análise cuidadosa da glândula tireoide e a determinação dos valores basais de TSH, T4 livre e anti-TPO. Os níveis de TSH e T4 livres devem ser medidos após três meses de terapia e, posteriormente, a cada seis meses.

\section{ABSTRACT}

It is presented here the case report of a 44 years old female patient admitted at the Intensive Care Unit (ICU) of the Hospital das Clínicas in Belo Horizonte/MG, with abdominal pain, nausea, vomiting, jaundice and fever, anorexia, loss of appetite, whose symptoms started 30 days before. She had a history of hypothyroidism being irregularly treated with T4 for the last five years, and her treatment was interrupted on the occasion the current symptoms appeared. She presented T3 (7.26 pg/ $\mathrm{mL}) \mathrm{VN}: 2.77$ to 5.07 ; TSH (0.214 IU/mL) VN: 0.465. Transaminases AST (977 U/L) VN: 15 to 46; ALT (795 U/L) VN:13 to 69; bilirubin total $(4.12 \mathrm{mg} / \mathrm{dL}) \mathrm{VN}$ : 0.2 to 1.3 ; direct bilirubin $(30 \mathrm{mg} / \mathrm{dL}) \mathrm{VN}$ : up to 0.25 . She was kept with no anti-thyroid and she started on propranolol at an up to $480 \mathrm{mg} / \mathrm{day}$ dosage. An MRI abdominal examination presented cholangiocarcinoma with free abdominal fluid, suggestive of being related to the inflammatory process. There was non-specific thickening of the gallbladder and bilateral pleural effusion. Serology examination for Hepatitis A, B, and C was negative. There was deterioration of the liver function and the high levels of thyroid hormones were kept. The patient suffered acute fulminant hepatic failure, and she was immediately submitted to hepatic transplant. After the procedure, the liver (explant) was sent for anatomopathological study; histopathologic findings included the presence of an extensive area of necrosis of the liver parenchyma. The remaining septa showed mononuclear inflammatory infiltrates (MNII). Therefore, it was concluded that chronic hyperthyroidism can exacerbate and perpetuate acute fulminant hepatic failure and the regular medical treatment, together with clinically routine laboratory examination could prevent severe complications, permitting a successful therapy in patients with hyperthyroidism.

Keywords: Thyroid, Hepatitis, Thyrotoxicosis, Liver, Inflammation. 


\section{REFERÊNCIAS}

1. Oppenheimer JH, Schwartz HL, Surks MI. Tissue differences in the concentration of triiodothyronine nuclear binding sites in the rat: liver, kidney, pituitary, heart, brain, spleen, and testis. Endocrinology. 1974;95(3):897-903.

2. Kim HJ, Kim BH, Han YS, Yang I, Kim KJ, Dong SH, et al. The incidence and clinical characteristics of symptomatic propylthiouracil-induced hepatic injury in patients with hyperthyroidism: a single-center retrospective study. Am J Gastroenterol. 2001;96(1):165-9.

3. Horst C, Rokos H, Seitz HJ. Rapid stimulation of hepatic oxygen consumption by 3,5-di-iodo-L-thyronine. Biochem J. 1989;261(3):945-50.

4. Liverini G, lossa S, Barletta A. Relationship between resting metabolism and hepatic metabolism: effect of hypothyroidism and 24 hours fasting. Horm Res. 1992;38(3-4):154-9.

5. Bayraktar M, Van Thiel DH. Abnormalities in measures of liver function and injury in thyroid disorders. Hepatogastroenterology. 1997;44(18):1614-8.

6. Fong TL, McHutchison JG, Reynolds TB. Hyperthyroidism and hepatic dysfunction. A case series analysis. J Clin Gastroenterol. 1992;14(3):240-4.

7. Huang MJ, Li KL, Wei JS, Wu SS, Fan KD, Liaw YF. Sequential liver and bone biochemical changes in hyperthyroidism: prospective controlled follow-up study. Am J Gastroenterol. 1994;89(7):1071-6.

8. Thompson P, Jr., Strum D, Boehm T, Wartofsky L. Abnormalities of liver function tests in tyrotoxicosis. Mil Med. 1978;143(8):548-51.

9. Gauna A, Gajst O, de Felice MC, Guillen C, Sartorio G, Viale F, et al. Modifications of serum hepatic enzymes in thyrotoxic patients with and without treatment. Medicina (B Aires). 1988;48(1):17-21.

10. Ichiki Y, Akahoshi M, Yamashita N, Morita C, Maruyama T, Horiuchi T, et al. Propylthiouracil-induced severe hepatitis: a case report and review of the literature. J Gastroenterol. 1998;33(5):747-50.
11. Russo MW, Galanko JA, Shrestha R, Fried MW, Watkins P. Liver transplantation for acute liver failure from drug induced liver injury in the United States. Liver Transpl. 2004;10(8):1018-23.

12. Xiao H, Zhuang W, Wang S, Yu B, Chen G, Zhou M, et al. Arterial embolization: a novel approach to thyroid ablative therapy for Graves' disease. J Clin Endocrinol Metab. 2002;87(8):3583-9.

13. Singer PA, Cooper DS, Levy EG, Ladenson PW, Braverman LE, Daniels G, et al. Treatment guidelines for patients with hyperthyroidism and hypothyroidism. Standards of Care Committee, American Thyroid Association. Jama. 1995;273(10):808-12.

14. Cooper DS. Antithyroid drugs. N Engl J Med. 2005;352(9):905-17.

15. Andrade VA, Gross JL, Maia AL. [Radioactive iodine therapy in Graves' hyperthyroidism]. Arq Bras Endocrinol Metabol. 2004;48(1):159-65.

16. Panzer C, Beazley R, Braverman L. Rapid preoperative preparation for severe hyperthyroid Graves' disease. J Clin Endocrinol Metab. 2004;89(5):2142-4.

17. Ashkar FS, Katims RB, Smoak WM, 3rd, Gilson AJ. Thyroid storm treatment with blood exchange and plasmapheresis. Jama. 1970;214(7):1275-9.

18. Schlienger JL, Faradji A, Sapin R, Blickle JF, Chabrier G, Simon C, et al. [Treatment of severe hyperthyroidism by plasma exchange. Clinical and biological efficacy. 8 cases]. Presse Med. 1985;14(23):1271-4.

19. Rangnekar AS, Ellerbec C, Durkalski V, McGuire B, Lee WM, Fontana RJ. Quality of life is significantly impared in long-term survivors of acute liver failure and particulary in acetaminophenoverdose patients Liver transplant. Liver Transpl. 2013; 19 (9): 991-1000.

20. Hambleton C, Buell J, Saggi B, Balart L, Shores NJ, Kandil E. Thyroid storm complicated by fulminant hepatic failure: case report and literature review. Ann Otol Rhinol Laryngol. 2013; 122 (11):679-82. 